\title{
DE LA PROSPECCIÓN SISTEMÁTICA AL LABORATORIO GIS EN La CANAl de NaVArRés (VAlencia)
}

La aplicación de métodos de prospección sistemática, a través de proyectos arqueológicos multidisciplinares, y la evaluación de sus resultados, ha permitido un gran avance en el análisis sobre la dimensión de la interacción humana con el medio ambiente a través del tiempo. Diversos proyectos de investigación centrados en este tipo de estudios a escala microrregional ponen de manifiesto como la valoración diacrónica de la transformación del paisaje y la actividad antrópica permite generar modelos valiosos para investigar la construcción del paisaje humano (Barton et al. 2012). Sirva de ejemplo el curso alto y medio del valle del río Serpis (o Alcoi) -Alicante-, donde este tipo investigación se ha canalizado mediante colaboraciones internacionales publicadas en los últimos 15 años (Barton et al. 1999; 2002; 2004; Bernabeu et al. 1999; Bernabeu et al. 2008). Fruto de estos trabajos son la reflexión sobre diversos aspectos metodológicos referidos al trabajo de campo de prospección, los aspectos tafonómicos que afectan a las colecciones superficiales (principalmente líticas) y la aplicación de criterios para su estudio, así como las propuestas para su valoración cronológica. Pero, principalmente, ha permitido esbozar una visión sobre la interacción humana con el paisaje desde el Paleolítico Medio hasta la época histórica enfocada en los valles del Serpis (Barton et al. 2004; Bernabeu et al. 2008; García Puchol y Aura Tortosa 2006).

El trabajo que presentamos se ha desarrollado en el marco del proyecto NSF The Emergence of Coupled Natural and Human Landscapes in the Western Mediterranean, segunda fase del proyecto Mediterranean Landsape Dynamics (MedLand), en el área comprendida por la actual comarca de La Canal de Navarres, situada al suroeste de la provincia de Valencia (fig. 1). Este espacio se convierte en una de las áreas elegidas para un programa de recogida de datos centrado en la prospección sistemática off site o patch-based (Barton et al. 2004; Dunnell 1992). Los datos relativos a la cultura material se combinan con la información espacial y ecológica, lo cual permite una comprensión dinámica de los mismos. La creación de estas bases de datos sobre determinados territorios resultará complementaria a la información arqueológica directa disponible resultado de las excavaciones.

En este sentido la elección de la Canal de Navarrés ha estado guiada por su potencial para explorar uno de los focos de interés principales en el proyecto: el impacto de la agricultura y la ganadería en la interacción humana sobre el paisaje y su evolución en el tiempo en las comarcas centrales valencianas. 


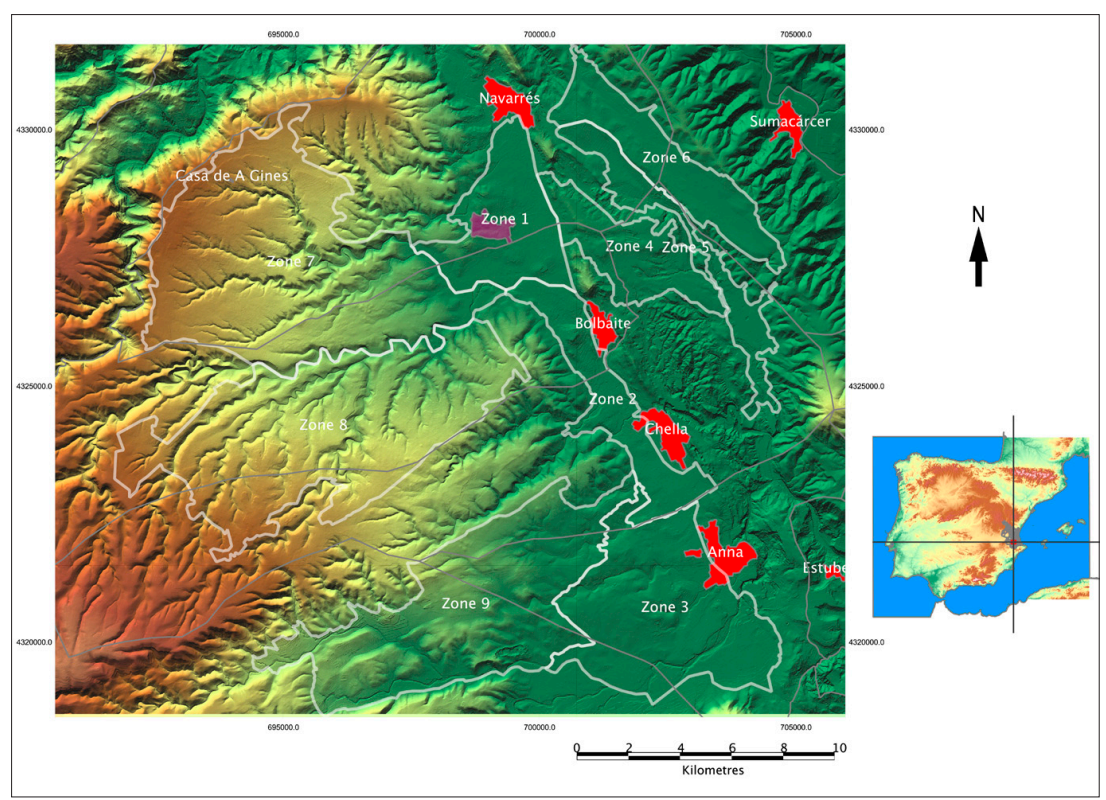

Fig. 1. Mapa de situación de la Canal de Navarrés y los principales yacimientos citados en el texto.

\section{MARCO ESPACIAL Y CONTEXTO ARQUEO- LÓGICO}

La Canal de Navarrés constituye un amplia cubeta semiendorreica colgada unos 200 metros sobre la red principal de drenaje en el área (el río Xúquer), localizada a unos $40 \mathrm{~km}$ del mar Mediterráneo (La Roca et al. 1999). De dirección NO-SE, se trata de un valle plano a $250 \mathrm{msnm}$ rodeado por las elevaciones del Macizo del Caroig (O) y la Serra de Sumacárcer (E) -al S del sistema Ibérico-. Está situado entre el corredor principal de conexión de la llanura costera valenciana con la meseta (el Corredor de Montesa), y el discurrir del río Xúquer en su salida hacia el mar. Este carácter semiendorreico propicia la presencia de zonas de marjal, alimentadas en gran medida por surgencias de agua subterránea, tales como la Albufera de Anna (Anna, Valencia) o las Fuentes y la Marjal de Navarrés (Navarrés, Valencia).

Los trabajos arqueológicos sistemáticos previos en el área se habían centrado precisamente en yacimientos situados en las inmediaciones de estos humedales durante los años 70 y 80 del pasado siglo. En el yacimiento de Las Fuentes (Navarrés) se realizaron unos sondeos en el interior del pequeño lago artificial del mismo nombre que propiciaron el descubrimiento de niveles con materiales atribuidos al Paleolítico Medio (Aparicio 1981). La buena preservación de la materia orgánica quedó reflejada en la documentación de restos de madera que se atribuyeron a un bosque fósil (pinus nigra) de elevada antigüedad, no bien precisada, en torno a unos 40.000 años (Aparicio 1981). Los trabajos arqueológicos en la Albufera d'Anna corresponden a unos sondeos realizados tanto en el interior de esta laguna artificial como en uno de sus laterales, aprovechando los trabajos de drenaje de la misma (Aparicio 1975, 1979). Entre los materiales recuperados destacan los geométricos, que permiten su atribución al Mesolítico final, y la presencia de cerámica a mano que apunta a la existencia de niveles neolíticos (Martí et al. 2009). El yacimiento que ha acaparado mayor atención arqueológica es sin duda La Ereta del Pedregal (Navarrés). Situado en las inmediaciones de Las Fuentes, los trabajos prolongados en el tiempo han permitido reconocer un singular yacimiento con niveles del Neolítico final, Calcolítico y de la Edad del Bronce (Juan Cabanilles 2008). Se trataría de un poblado construido en la orilla del humedal de $\mathrm{Na}$ varrés que destaca por presentar construcciones en piedra atribuidas a espacios domésticos y a una posible muralla. Al mismo tiempo, la presencia de depósitos de turba ha sido aprovechada para la realización de dos sondeos palinológicos cuyos resultados permiten obtener una imagen de la historia de la vegetación en el área durante los últimos 20.000 años (Carrión y Van Geel 1999). 


\section{METODOLOGÍA}

Hemos escogido para nuestro análisis el amplio espacio comprendido entre el fondo de los valles y el pie de monte, siguiendo una estrategia focalizada en la detección de las actividades antrópicas desde una perspectiva diacrónica en el paisaje de la Canal de Navarrés. El área ha quedado dividida en nueve zonas que no incluyen el casco urbano de los municipios afectados: Anna, Chella, Bolbaite y Navarrés. Cada una de estas grandes zonas, de tamaño similar, se ha subdividido en una serie de sectores, delimitados por los principales caminos de acceso y/o barrancos. Se ha seguido una estrategia aleatoria para la selección de un sector de cada uno de las zonas considerados utilizando el software R (R Core Team 2013). Las unidades de prospección (subsectores) dentro de estos sectores se definen a través de una serie de unidades espaciales, en nuestro caso hemos considerado las parcelas recogidas en el catastro de la Generalitat Valenciana. En el caso de parcelas de gran tamaño se ha procedido a su subdivisión reflejada a través de un subíndice numérico. De cada una de estas unidades se recogen los datos relativos a la cultura material pero también información descriptiva sobre el terreno (visibilidad, uso del suelo, grado de transformación). Hemos comprobado la versatilidad en el uso del iPad para la recogida de datos de campo mediante una app (CartoMobile) que permite visualizar las ortofotos y el mapa del catastro de las áreas prospectadas. Una sencilla base de datos permite guardar los datos de campo. Los GPS Garmin manuales se han utilizado para documentar los tracks individuales efectuados. De forma paralela se ha registrado la informacion en dispositivos Android con la app OsmAnd.

En el laboratorio se ha llevado a cabo la clasificación y descripción de los materiales y su implementación en una base datos (Filemaker). La plataforma GIS (QgisDevelopment Team 2014) posibilitará la asociación entre los datos espaciales y el registro material. De otro lado, los gráficos e índices presentados en el apartado de resultados se han elaborado mediante el software $\mathrm{R}$ (R Core Team 2013).

\section{RESULTADOS}

A lo largo de esta campaña de prospección (junio 2014) se ha intervenido en siete de las nueve zonas en los que ha quedado dividida el área (zonas 1, 2, 3, 5, 6, 7 y 9). De éstas se ha procedido a prospectar el $33 \%$ de uno de los sectores considerados, con la excepción dde la zona 7, donde el área cubierta ha sido menor. Los materiales recogidos durante la prospección se identifican mediante una sigla que corresponde al zona, sector y parcela del catastro (ep.e., 9-6-3, 3-3-29, etc). En total
Fig. 2. Tabla donde quedan reflejados los subsectores prospectados y los subsectores con hallazgos.

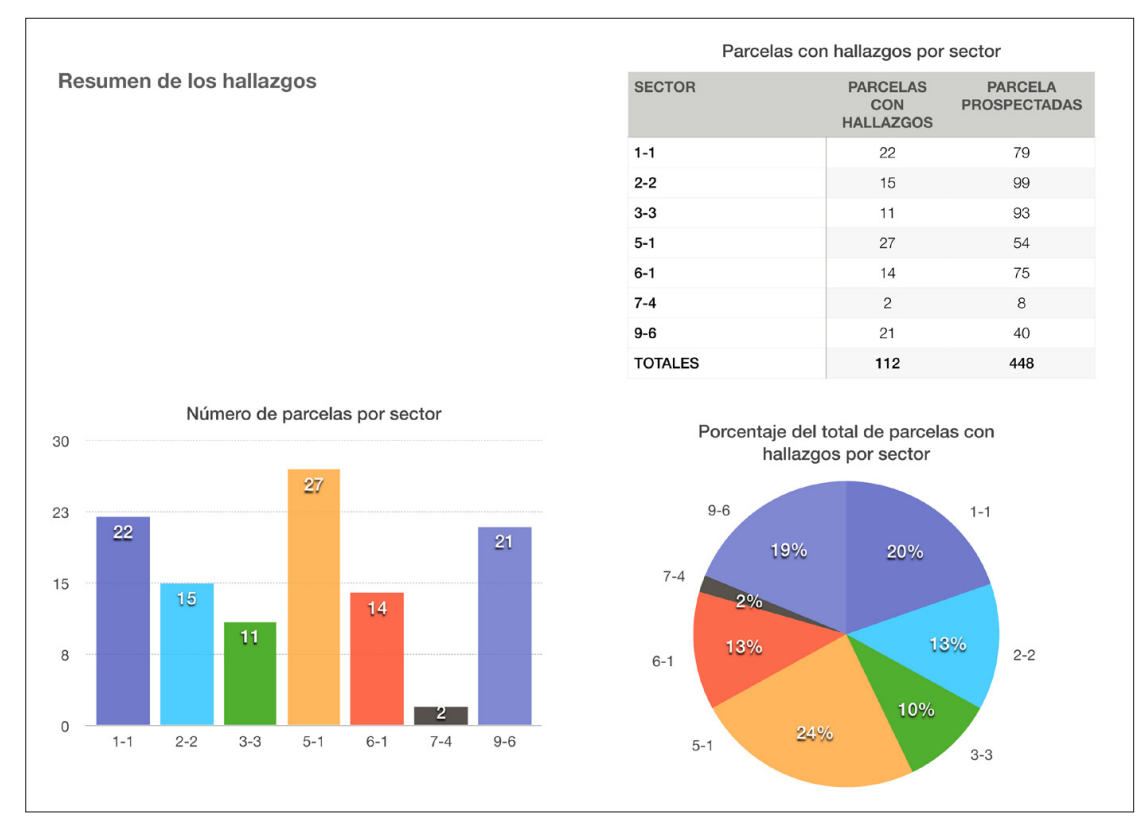




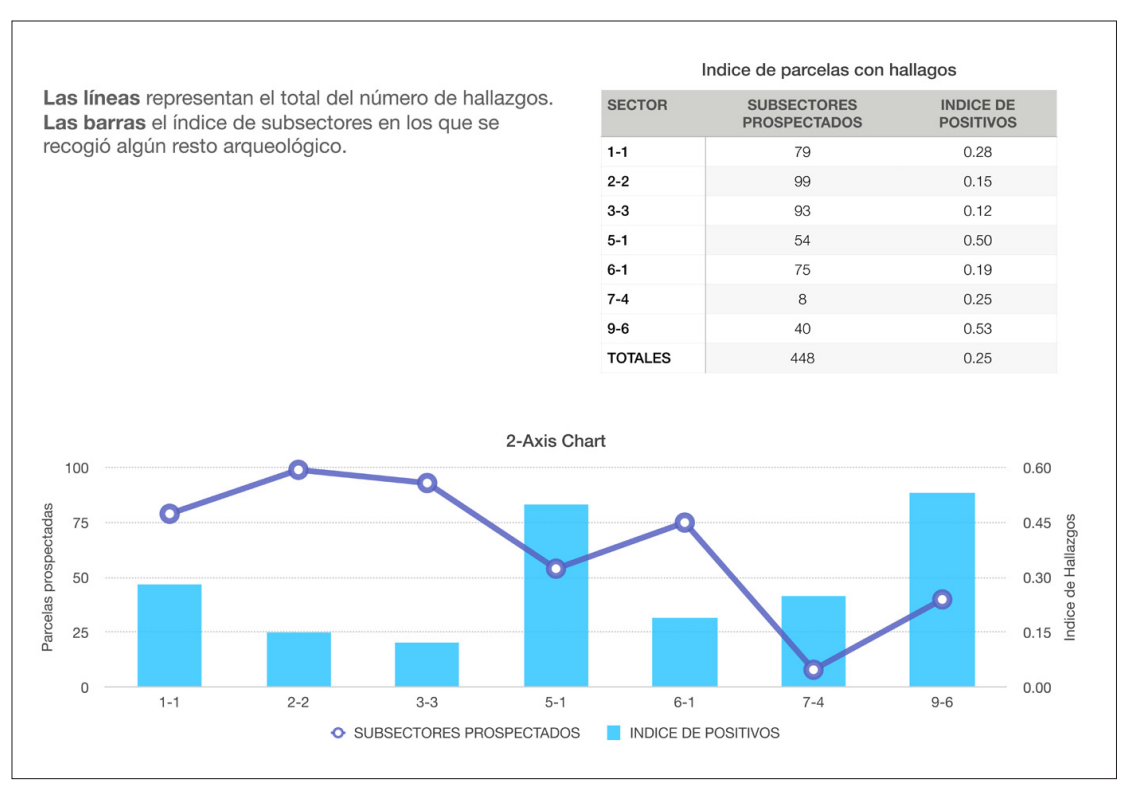

Fig. 3. Gráfico donde se representa el Índice de positivos.

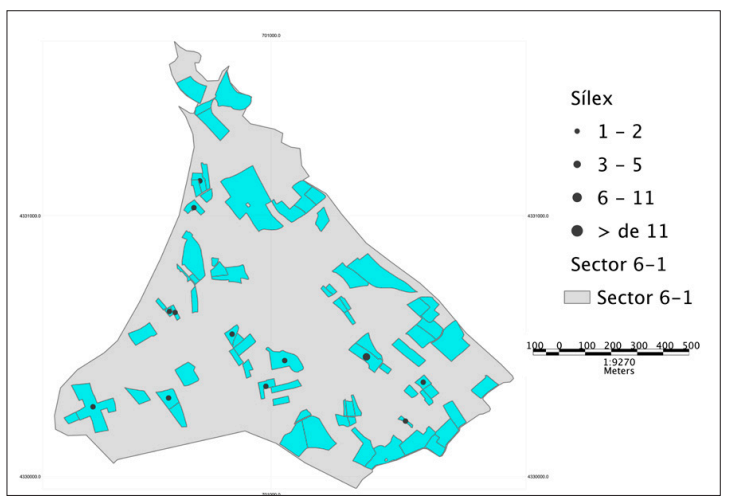

Fig. 4. Mapa de la zona 6 con indicación de la densidad de hallazgos por parcela.

se ha prospectado un área de 258,9 ha que corresponde a 448 parcelas (unidad mínima de prospección). El detalle por cada uno de los sectores queda reflejado en las figura 2.

La figura 2 muestra además los sectores con hallazgos. Como podemos observar su repartición es desigual, de forma que las zonas 1, 5 y 9 concentran el mayor número de parcelas con hallazgos. La figura 3 ilustra sobre los hallazgos por parcela, resultado de dividir el número de parcelas con hallazgos por el total de parcelas del sector (Índice de positivos: IP). El índice mayor se identifica con las zonas 5 y 9 mientras que las zonas 2 y 3 ofrecen un índice bajo.
Los hallazgos principalmente corresponden a piedra tallada, sobre todo sílex, y en un número testimonial a caliza y cuarcita. Se han observado algunas concentraciones particulares (5-1-181) entre las que destacan los fragmentos indeterminados y los restos de talla (principalmente lascas y alguna lámina/laminita), y en menor proporción algún útil retocado. La figura 4 presenta la distribución de los hallazgos líticos en la zona 6 , sector 1 . Un reducido número de objetos permite hacer una primera aproximación sobre su cronología, principalmente algunos ítems identificados como proyectiles u otros útiles cuyo rango cronológico puede determinarse (puntas de flecha, raspadores, buriles, laminitas de dorso, dientes de hoz). Nos limitaremos en estas páginas a apuntar esta relación, que indicaría la presencia de materiales desde el Pleistoceno Superior final hasta el Holoceno medio, a la espera de procesar los datos en base a un sistema de rangos que permita dilucidar estadísticamente el grado de asignación tal como hemos planteado en otros trabajos (Barton et al. 1999; 2001; 2004; Bernabeu et al. 1999). Podemos así concluir que la documentación de materiales líticos abarca un rango cronológico desde el Paleolítico Superior Final a la Edad del Bronce, con algunas concentraciones puntuales y, sobre todo, ejemplos de materiales dispersos.

La cerámica recuperada ha sido atribuida en gran medida a momentos históricos (época ibérica, romana y medieval). Únicamente se han clasificado 3 fragmentos de cerámica a mano (posiblemente de la Edad del Bronce). 


\section{CONCLUSIÓN}

Los resultados preliminares que presentamos en este trabajo reflejan el interés de contemplar este tipo de estudios off site para la obtención de una visión global sobre las formas de ocupación del territorio. La metodología empleada (uso del iPad y app GIS) ha resultado de gran utilidad por su versatilidad para la recogida de datos de campo. El objetivo contempla la implementación de esta información en una plataforma GIS que integrará los datos arqueológicos, ecológicos y geográficos para la obtención de modelos diacrónicos sobre la interacción humana sobre el medio ambiente. El análisis de la colección de materiales permite albergar expectativas sobre la obtención de información en entornos que han sufrido el impacto de la acción humana en forma de explotaciones agrícolas más o menos intensa. Si el grado de transformación determinará la relación de los materiales con el paisaje actual y pretérito, no es menos cierto que la presencia o ausencia de datos introducen señales válidas para su interpretación de histórica.

Las evidencias de ocupación humana en la Canal de Navarrés se remontan al Paleolítico medio. Hasta la fecha, los vestigios de ocupaciones al aire libre de tres yacimientos han acaparado los trabajos sistemáticos, de mayor o menor alcance, realizados. La información arqueológica publicada es en general escasa y refiere en la mayoría de los casos hallazgos superficiales al aire libre o bien en algunas cuevas y abrigos. De los datos manejados se desprende el potencial interés de un área donde es posible reconocer lugares de ocupación de los últimos caza-recolectores y, puntualmente, la existencia de materiales atribuibles al Neolítico antiguo (cerámica cardial en Cueva de la Bellota, Chella). La prospección efectuada ofrece nuevos datos para valorar la intensidad de la ocupación desde una perspectiva diacrónica y también sobre la distribución de los mismos en el espacio. El avance en la evaluación de la información contribuirá a la reflexión sobre algunos de los puntos aquí esbozados.

Oreto García Puchol Programa Ramón y Cajal Dept. Prehistòria i Arqueologia Universitat de València oreto.garcia@uv.es

Joan Bernabeu Aubán Agustín Diez Castillo SAlvador Pardo Gordó Dept. Prehistòria i Arqueologia Universitat de València

\author{
Juan.Bernabeu@uv.es \\ agustin.diez@uv.es \\ salvador.pardo@uv.es \\ C. Michael Barton \\ School of Human Evolution and Social Change \\ College of Liberal Arts \\ Arizona State University \\ Center for Social Dynamics and Complexity \\ Michael.Barton@asu.edu
}

\section{BIBLIOGRAFÍA}

APARICIO, J. (1975): Los yacimientos prehistóricos de la Albufera de Anna (Valencia), XIII Congreso Nacional de Arqueología (Huelva, 1973), Zaragoza, 191-198.

APARICIO, J. (1979): El Mesolítico en Valencia y en el Mediterráneo occidental, Valencia.

BARTON, C. M.; BERNABEU, J.; AURA, J. E.; GARCÍA PUCHOL, O. (1999): Landscape dynamics and socioeconomic change: an example from the Polop Alto valley, American Antiquity 64 (4), 609-634. DOI: http://dx.doi.org/10.2307/2694208

BARTON, C. M.; BERNABEU, J.; AURA, J.E.; GARCÍA PUCHOL, O.; LA ROCA, N. (2002): Dynamic landscapes, artifact taphonomy, and landuse modeling in the western Mediterranean, Geoarchaeology 17 (2), 155-190. DOI: http://dx.doi.org/10.1002/gea.10008

BARTON, C. M.; BERNABEU, J.; GARCIA, O.; SCHMICH, S.; MOLINA, LL. (2004): Long-term socioecology and contingent landscapes, Journal of Archaeological Method and Theory 11 (3), 253-295.

DOI: http://dx.doi.org/10.1023/B:JARM.0000047315.57162.b7

BARTON, C. M.; ULLAH, I. I. T.; BERGIN, S. M.; MITASOVA, H.; SARJOUGHIAN, H. (2012): Looking for the future in the past: long-term change in socioecological systems, Ecological Modelling 241, 42-53. DOI: http://dx.doi.org/10.1016/j.ecolmodel.2012.02.010

BERNABEU, J.; BARTON, C. M.; GARCÍA PUCHOL, O.; LA ROCA, N. (1999): Prospecciones sistemáticas en el valle del Alcoi (Alicante): primeros resultados, Arqueología Espacial 21, 29-64.

BERNABEU, J.; MOLINA, LL.; OROZCO, T.; DIEZ, A.; BARTON, C. M. (2008): Los valles del Serpis (Alicante): 20 años de trabajo de campo, IV Congreso del Neolítico Peninsular (M.S. Hernández Pérez, J.A. Soler Díaz, J.A. López Padilla, coords.), Alicante, 50-57.

CARRIÓN, J. S.; VAN GEL, B. (1999): Fine-resolution Upper Weichselian and Holocene palynological record from Navarrés (Valencia, Spain) and a discussion about factors of Mediterranean forest succession, Review of Paleobotany and Palynology 106, 209-236. DOI: http://dx.doi.org/10.1016/S0034-6667(99)00009-3 
DUNNELL, R. C. (1992): The notion of site, Space, Time, and Archaeological Landscapes (J. Rossignol, L. Wandsnider, eds.), Plenum, New York, 21-41.

DOI: http://dx.doi.org/10.1007/978-1-4899-2450-6_2

GARCÍA PUCHOL, O.; AURA TORTOSA, J. E. (2006): El Abric de la Falguera (Alcoi, Alacant). 8.000 años de ocupación humana en la cabecera del río Alcoi. MARQ Diputación de Alicante, Caja de Ahorros del mediterráneo y Ajuntament de Alcoi, vol. 1.

JUAN CABANILLES, J. (2008): El utillaje de piedra tallada en la Prehistoria reciente valenciana. Aspectos tipológicos, estilísticos y evolutivos, Serie de Trabajos Varios del SIP 109, Valencia.

LA ROCA, N.; FUMANAL, M. P.; MARTÍNEZ GALLEGO, J. (1996): Evolución cuaternaria del drenaje en un corredor intramontano: La Canal de Navarrés (Valencia, Spain), IV Reunión de Geomorfología. Sociedad Española de Geomorfología (A. Grandal d'Anglade, J. Pagés Valcarlos, eds.), O Castro (A Coruña), Cuadernos do Laboratorio Xeolóxico de Laxe 21, 445-455.

MARTÍ, B.; AURA, J. E.; JUAN CABANILLES, J.; GARCÍA PUCHOL O.; FERNÁNDEZ, J. (2009): El mesolítico Geométrico de tipo "Cocina” en el País Valenciano, El mesolítico Geométrico en la Península Ibérica (P. Utri1la, L. Montes, eds.), Actas de la Reunión de Jaca, 2008, Universidad de Zaragoza, Monografías Arqueológicas 44, 205-258.

R CORE TEAM (2013): A language and environment for statistical computing, $\mathrm{R}$ foundation for Statistical Computing, Viena. URL://www.R-project.org 